\title{
Observation of two-magnon bound states in the two-leg ladders of $(\mathrm{Ca}, \mathrm{La}){ }_{14} \mathrm{Cu}_{24} \mathrm{O}_{41}$
}

\author{
M. Windt, ${ }^{1}$ M. Grüninger, ${ }^{1}$ T. Nunner,${ }^{2}$ C. Knetter,${ }^{3}$ K. Schmidt,${ }^{3}$ G.S. Uhrig, ${ }^{3}$ T. Kopp,${ }^{2}$ A. Freimuth,${ }^{1}$ \\ U. Ammerahl, ${ }^{1,5}$ B. Büchner, ${ }^{1,4}$ and A. Revcolevschi ${ }^{5}$ \\ ${ }^{1}$ II. Physikalisches Institut, Universität zu Köln, 50937 Köln, Germany \\ ${ }^{2}$ Experimentalphysik VI, Universität Augsburg, 86135 Augsburg, Germany \\ ${ }^{3}$ Institut für Theoretische Physik, Universität zu Köln, 50937 Köln, Germany \\ ${ }^{4}$ II. Physikalisches Institut, RWTH-Aachen, 52056 Aachen, Germany \\ ${ }^{5}$ Laboratoire de Chimie des Solides, Université Paris-Sud, 91405 Orsay Cédex, France
}

(March 20, 2001)

\begin{abstract}
Phonon-assisted 2-magnon absorption is studied at $\mathrm{T}=4 \mathrm{~K}$ in the spin-1/2 2-leg ladders of $\mathrm{Ca}_{14-x} \mathrm{La}_{x} \mathrm{Cu}_{24} \mathrm{O}_{41}(x=5$ and 4 ) for $E \| c$ (legs) and $E \| a$ (rungs). Two peaks at about 2140 and $2800 \mathrm{~cm}^{-1}$ reflect van-Hove singularities in the density of states of the strongly dispersing 2-magnon singlet bound state, and a broad peak at $\approx 4000 \mathrm{~cm}^{-1}$ is identified with the 2-magnon continuum. Two different theoretical approaches (Jordan-Wigner fermions and perturbation theory) describe the data very well for $J_{\|} \approx 1050-1100 \mathrm{~cm}^{-1}, J_{\|} / J_{\perp} \approx 1-1.1$. A striking similarity of the high-energy continuum absorption of the ladders and of the undoped high $T_{c}$ cuprates is observed.

PACS numbers: 74.72.Jt, 78.30.-j, 75.40.Gb, 75.10.Jm
\end{abstract}

Low-dimensional quantum spin systems display a fascinating variety of low-energy excitations. Prominent examples are the fractional quantum states of onedimensional (1D) chains, the $S=1 / 2$ spinons, or the variety of bound states in gapped spin liquids such as, e.g., dimerized chains or even-leg ladders. In $2 \mathrm{D}$, the magnetic excitations of the undoped high- $T_{c}$ cuprates are usually discussed in more conventional terms, namely renormalized spin waves. But these fail to describe the shortwavelength high-energy excitations probed by optical 2magnon-plus-phonon absorption. 1 The nature of incoherent high-energy excitations is currently under intensive debate.2

In this light, the study of other cuprates with related topologies is very instructive. As far as high-energy excitations are concerned, the $\mathrm{Cu}_{2} \mathrm{O}_{3}$ 2-leg ladders realized in $(\mathrm{Ca}, \mathrm{La}){ }_{14} \mathrm{Cu}_{24} \mathrm{O}_{41}$ (Ref. 6) provide a bridge between $1 \mathrm{D}$ physics and the $2 \mathrm{D} \mathrm{CuO}_{2}$ layers. Antiferromagnetic $S=1 / 2$ 2-leg Heisenberg ladders are represented by the Hamiltonian

$\mathcal{H}=\sum_{i}\left\{J_{\|}\left(\mathbf{S}_{1, i} \mathbf{S}_{1, i+1}+\mathbf{S}_{2, i} \mathbf{S}_{2, i+1}\right)+J_{\perp} \mathbf{S}_{1, i} \mathbf{S}_{2, i}\right\}$,

where $J_{\perp}$ and $J_{\|}$denote the rung and leg couplings, respectively. For $J_{\|}=0$ one can excite local rung singlets to rung triplets which become dispersive on finite $J_{\|}$. For $J_{\perp}=0$ the $S=1$ chain excitations decay into asymptotically free $S=1 / 2$ spinons. An intuitive picture of the "magnons" (elementary triplets) for $J_{\perp}, J_{\|} \neq 0$ can be obtained from both limits: the elementary excitations are either dressed triplet excitations or pairs of bound spinons with a finite gap $\Delta$ as long as $J_{\perp}>0$. In a gapped system it is particularly interesting whether bound states occur. Theoretical studies of 2-leg ladders show that bothsinglet and triplet 2-magnon bound states always exist 12 However, in the $S=1 / 2$ copper oxides their experimental observation is a difficult task. Inelastic neutron scattering directly probes the spin gap, but cannot determine the high-energy excitations to a suf. ficient extent due to the large exchange interactions. 13 Magnetic Raman scattering 14 is restricted to $k=0$ excitations, but for the relevant values of $J_{\|} / J_{\perp}$ the singlet bound state appears only for finite wave vector (see Fig. 3 and Ref. 12). In this case, optical spectroscopy is the ap propriate tool.10 Two-magnon-plus-phonon absorption 15 is able to probe magnetic excitations throughout the entire Brillouin zone (BZ), since the simultaneous excitation of a phonon takes care of momentum conservation.

In this paper, we present optical conductivity data of $\mathrm{Ca}_{14-x} \mathrm{La}_{x} \mathrm{Cu}_{24} \mathrm{O}_{41}$ and identify the 2-magnon singlet bound state by two peaks reflecting van-Hove singularities in the density of states (DOS) of the bound state. Our theoretical results for bound states in the experimentally relevant parameter range are based on turg different approaches, namely Jordan-Wigner fermions 16 and perturbation expansion about the strong dimer coupling limit up to 13th order using unitary transformations.17.8 Both yield an excellent description of the optical data for $J_{\|} \approx 1050-1100 \mathrm{~cm}^{-1}$ and $J_{\|} / J_{\perp} \approx 1-1.1$.

Single crystals with $x=5$ and 4 were orown by the travelling solvent floating zone method 19 Their single phase structure and stoichiometry have been verified by $\mathrm{x}$-ray, energy dispersive x-ray and thermogravimetric analyses 19 The La content $x$ determines the average oxidation state of $\mathrm{Cu}$. A nominally undoped sample is obtained for $x=6$, which probably is beyond the La solubility limit. 19 Single phase crystals could only be synthesized for $x \leq 5$. The samples with $x=5$ and 4 on average contain $n=1 / 24$ and $2 / 24$ holes per $\mathrm{Cu}$, respectively. But x-ray absorption data show that at least for $n \leq 4 / 24$ the holes are located within the chains, 20 which agrees with previous considerations.21 22 Thus we consider the lad- 


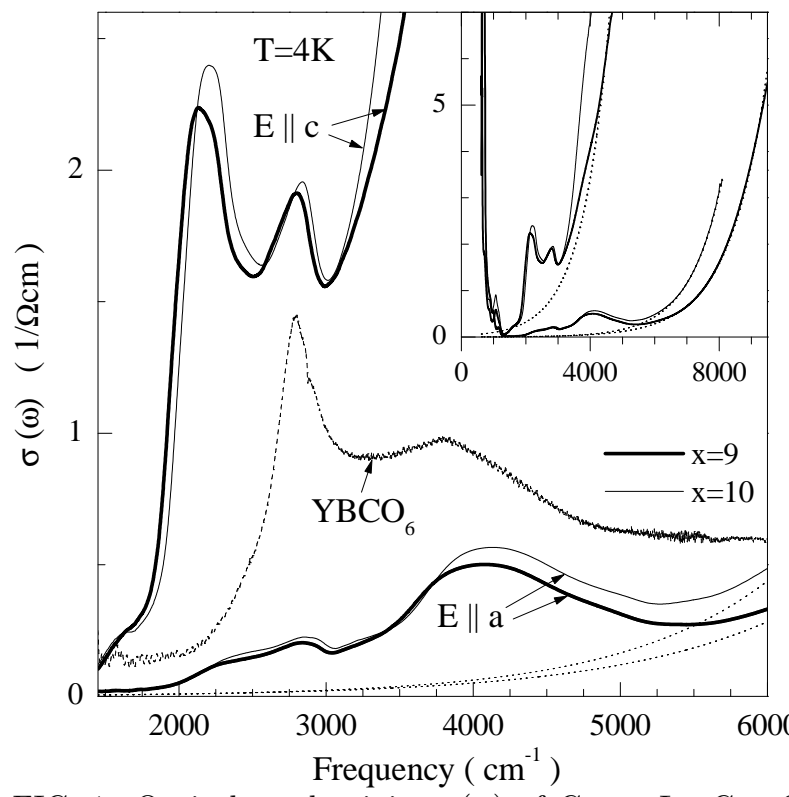

FIG. 1. Optical conductivity $\sigma(\omega)$ of $\mathrm{Ca}_{14-x} \mathrm{La}_{x} \mathrm{Cu}_{24} \mathrm{O}_{41}$ (thick lines: $x=5$; thin solid lines: $x=4$ ) for $E \| c$ (legs) and $E \| a$ (rungs) at $4 \mathrm{~K}$. Dashed: Bimagnon-plus-phonon absorption of $\mathrm{YBa}_{2} \mathrm{Cu}_{3} \mathrm{O}_{6}$ is given for comparison. ${ }^{1}$ Inset: $(\mathrm{Ca}, \mathrm{La})_{14} \mathrm{Cu}_{24} \mathrm{O}_{41}$ data on a larger scale. Dotted: estimate of the electronic background (exponential fits for $\omega>6500$ $\mathrm{cm}^{-1}$ for $E \| a ; \omega>4500 \mathrm{~cm}^{-1}$ for $\left.E \| c\right)$.

ders to be undoped which is supported by the similarity of our results for $x=5$ and $x=4$.

The optical conductivity $\sigma(\omega)$ was determined by collecting both transmission 23 and reflection data between 500 and $12000 \mathrm{~cm}^{-1}$ on a Fourier spectrometer. In Fig. 1 we display $\sigma(\omega)$ of $\mathrm{Ca}_{14-x} \mathrm{La}_{x} \mathrm{Cu}_{24} \mathrm{O}_{41}$ at $\mathrm{T}=4 \mathrm{~K}$ for polarization of the electrical field parallel to the legs, $E \| c$, and to the rungs, $E \| a$. Phonon and multi-phonon absorption dominates $\sigma(\omega)$ below $\approx 1300 \mathrm{~cm}^{-1}$ (see inset). A steep increase of the electronic background, probably due to interband excitations of charge-transfer type, is observed above $6000 \mathrm{~cm}^{-1}$ for $E \| a\left(3000 \mathrm{~cm}^{-1}\right.$ for $E \| c$ ). Our analysis focuses on the two peaks between 2000 and $3000 \mathrm{~cm}^{-1}$ and the broad feature at $4000 \mathrm{~cm}^{-1}$. Also plotted in Fig. 1 is $\sigma(\omega)$ of $\mathrm{YBa}_{2} \mathrm{Cu}_{3} \mathrm{O}_{6}$ (Ref. 1), a typical example of the 2-magnon-plus-phonon absorption spectrum of the undoped 2D cuprates 15 This comparison gives a first motivation to interpret the peaks in $\sigma(\omega)$ of the ladders as magnetic excitations. Note that in the ladders both the exchange constants and the relevant $\mathrm{Cu}$ $\mathrm{O}$ bond stretching phonon frequencies are comparable to those found in the $2 \mathrm{D}$ cuprates. Since the exchange coupling in the chains is much smaller, $J_{\text {chain }} \approx-14 \mathrm{~cm}^{-1}$ (Ref. 22), their magnetic excitations do not contribute to $\sigma(\omega)$ in this frequency range. Reducing the La content $x$ from 5 to 4 causes a slight blue-shift of the magnetic absorption that is opposite to the red-shift of the electronic background. We attribute the blue-shift to an increase of the exchange constants caused by the reduction of the lattice parameters ( $\mathrm{Ca}$ is smaller than La), and the red-

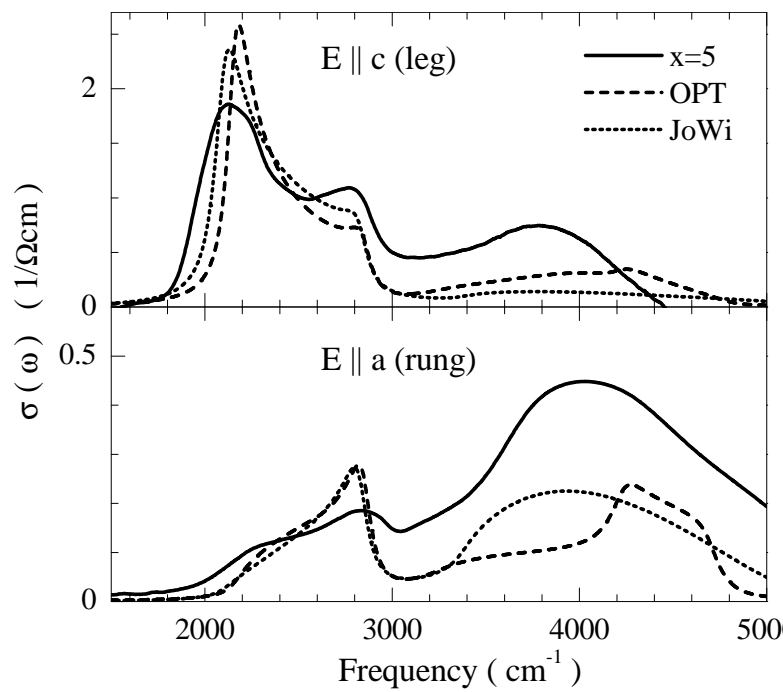

FIG. 2. Comparison of experiment (solid lines for $x=5$ ) and theory for $J_{\perp}=J_{\|}=1050 \mathrm{~cm}^{-1}$ (OPT: optimized perturbation) and $1100 \mathrm{~cm}^{-1}$ (JoWi: Jordan-Wigner fermions), with $\omega_{p h}=600 \mathrm{~cm}^{-1}$. The exponential fits of the electronic background have been subtracted from the experimental data (dotted lines in Fig. 1).

shift of the background to an increased hole density in the chains.

Since spin is conserved, infrared absorption is sensitive to $\Delta S=0$, singlet excitations, e.g., the excitation of two $S=1$ magnons coupled to $S_{\mathrm{tot}}=0$. Direct excitation of two magnons is Raman active 14 but infrared forbidden due to the inversion symmetry on a $180^{\circ} \mathrm{Cu}-\mathrm{O}-\mathrm{Cu}$ bond. We can effectively avoid this selection rule by simultaneously exciting a $\mathrm{CH}_{-} \mathrm{O}$ bond stretching phonon that breaks the symmetry. 15 Hence the lowest order infraredactive magnetic absorption is a 2-magnon-plus-phonon process. The low values of $\sigma(\omega) \lesssim 2 \Omega^{-1} \mathrm{~cm}^{-1}$ indeed indicate a weak higher-order absorption process.

The 2-magnon-plus-phonon contribution to $\sigma(\omega)$ was evaluated by Jurecka and Brenig 10 in the extreme case of strong rung coupling $J_{\|} \rightarrow 0$. They found a single sharp peak reflecting the singlet bound state. In order to gain quantitative control over the experimentally relevant coupling regime of $J_{\|} / J_{\perp} \approx 1$ we use two different approaches to calculate the dynamical 4-point spin correlation function, namely Jordan-Wigner fermions and perturbation theory 24 In the former, we make use of the Jordan-Wigner transformation 16 to rewrite the spins as fermions with a long-ranged phase factor. Expanding the phase factor yields new interaction terms between the fermions which we treat in RPA (random phase approximation). The second approach is perturbative in nature. It is performed by a continuous unitary transformation. 17 . 8 The 1-particle energies and the 2-particle bound state energies are extrapolated by standard techniques (Padé and Dlog-Padé); the spectral densities are computed by optimized perturbation 25 Details will be given elsewhere. The magnon (elementary triplet) 


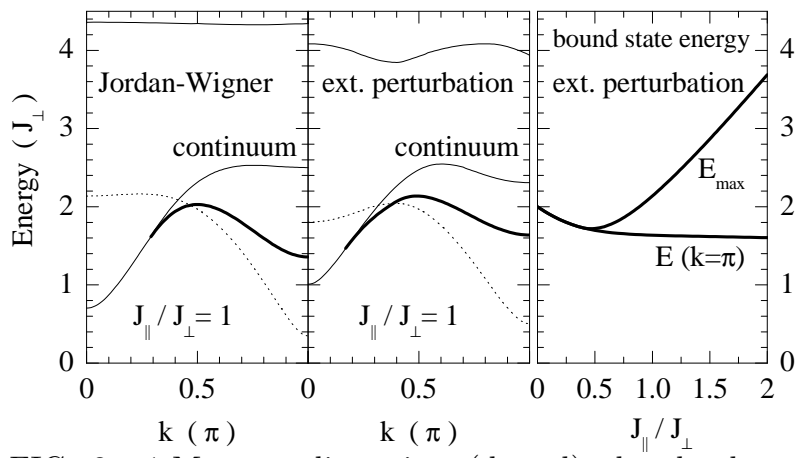

FIG. 3. 1-Magnon dispersion (dotted), band edges of the 2-magnon continuum (solid), and bound singlet dispersion (thick lines) for $J_{\|} / J_{\perp}=1$ obtained from Jordan-Wigner fermions (left) and extrapolated perturbation (mid panel). Right: energy of the van-Hove singularities of the bound singlet at $k=\pi$ and at the dispersion maximum as a function of $J_{\|} / J_{\perp}$.

dispersion, the 2-particle continuum and the dispersion of the singlet bound state obtained for $J_{\perp}=J_{\|}$are given in the two left panels of Fig. 3. The results of the two techniques agree well even though there is a quantitative discrepancy $(\lesssim 10 \%)$ in some energies (dispersion at $k=0$ and $\pi$, bound state energy). The magnon dispersion of extrapolated perturbatipn (mid panel) reproduces previous results very well.26 27 We focus on the singlet bound state which shows a considerable dip at $k=\pi$. This has gone unnoted thus far because it occurs only for $J_{\|} / J_{\perp} \gtrsim 0.5$ (see right panel in Fig. 3 and Ref. 11).

To compare with experiment we consider the effect of the phonons. The total spectral weight is obtained by taking into account a dependence of the exchange constants on the external electric field $\mathbf{E}$ and the displacements of $\mathrm{O}$ ions $\mathbf{u}, J_{\|, \perp} \equiv J_{\|, \perp}(\mathbf{E}, \mathbf{u})$ (Refs. 15, 1). The phonons modulate the intersite hopping and the on-site energies on both $\mathrm{Cu}$ and $\mathrm{O}$ sites. We expand $J(\mathbf{E}, \mathbf{u})$ to order $d^{2} J / d \mathbf{u} d \mathbf{E}$ which entails the coupling of a photon to a phonon and two neighboring spins. This determines how to integrate the spin response in the BZ. Here, the weight factor is a mixture of $\sin ^{4}(k / 2)$ (Ref. 28) and of an isotropic (k-jndependent) form factor, where the former dominates 29 For simplicity we consider a pure form factor $\sin ^{4}(k / 2)$ and Einstein phonons with $\omega_{p h}=600 \mathrm{~cm}^{-1}$ as is common for the cuprates. Our findings depend only very weakly on the precise phonon energy.

In Fig. 2 we compare the theoretical results with the experimental data. For the former an artificial broadening of $J_{\perp} / 20$ is used. For the latter, the magnetic part of $\sigma(\omega)$ was obtained by subtracting the electronic background (dotted lines in Fig. 1). For $E \| a$, the background was determined unambiguously by an exponential fit for $\omega>6500 \mathrm{~cm}^{-1}$. The $\sigma_{a}(\omega)$ curves for $x=5$ and 4 nearly coincide after subtraction of the background. This corroborates the assumption that the ladders are undoped. For $E \| c$, the measurable range of transmission was limited to below $\approx 4500 \mathrm{~cm}^{-1}$ by the higher absorption, and the precise shape and spectral weight of the highest peak at $\approx 4000 \mathrm{~cm}^{-1}$ cannot be determined unambiguously.

The two peaks at 2140 and $2800 \mathrm{~cm}^{-1}(x=5)$ can be identified with the $1 \mathrm{D}$ van-Hove singularities in the DOS of the singlet bound state. The lower peak corresponds to the singlet energy at the BZ boundary, $E(k=\pi)$, the higher one to the maximum of the singlet dispersion $E_{\max }$ at about $k \approx \pi / 2$ (see Fig. 3). These two energies determine the two free magnetic parameters $J_{\perp}$ and $J_{\|}$. The experimental $\sigma(\omega)$ is well described by both theories for $J_{\|} \approx 1050-1100 \mathrm{~cm}^{-1}$ and $J_{\|} / J_{\perp} \approx 1$ (see Fig. 2) providing an unambiguous identification of the experimental features The quantitative analysis can be pushed a step further 30 based on the values of $E_{\max }$ and $E(k=\pi)$ computed by extrapolated perturbation (right panel of Fig. 3). The strong dependence of $E_{\max }$ on $J_{\|} / J_{\perp}$ allows to pinpoint the exchange constants, yielding the same value for $J_{\|} \approx 1050 \mathrm{~cm}^{-1}$ and a slightly larger ratio of $J_{\|} / J_{\perp} \approx 1$.1. Our interpretation of the experimental features is confirmed by the good agreement between theory and experiment concerning the line shape of the bound states in $\sigma(\omega)$. Excellent justification for this interpretation is also provided by the selection rule stemming from reflection symmetry about the $a$ axis (RSa). Both theories show that the bound singlet at $k=\pi$ is even under $\mathrm{RS} a$. But the excitations at $k=\pi$ are odd under RS $a$ for $E \| a$ and even for $E \| c$. Thus the weight of the bound state varies as $(k-\pi)^{2}$ for $E \| a$ whereas it is prevailing for $E \| c$. This explains the low spectral weight of the lower peak for $E \| a$. It is reduced to a weak shoulder.

Shortcomings of the theory are the overestimation of the spectral weight of the $2800 \mathrm{~cm}^{-1}$ peak for $E \| a$ and that the onset of $\sigma(\omega)$ around $2000 \mathrm{~cm}^{-1}$ is sharper than observed experimentally. However, the agreement is better than one may have expected since we neglected both the frustrating coupling between neighboring ladders and the ring exchange. A finite inter-ladder coupling will produce a dispersion of the bound state along $k_{a}$ and thereby broaden the features in $\sigma(\omega)$, which can explain a smearing out of the onset at $2000 \mathrm{~cm}^{-1}$. Without the ring exchange, the analysis of experimental data of various other techniques suggested a larger leg coupling $J_{\|} / J_{\perp} \gtrsim 1.5$ (for a detailed discussion, see Ref. 26). One finds a 1-magnon gap of $\Delta \approx 280 \mathrm{~cm}^{-1}$ and a dispersion extending up to $\approx 1550 \mathrm{~cm}^{-1}$ (Ref. 13). For $J_{\|} / J_{\perp}=1$ the dispersion extends from $\Delta \approx 0.5 J_{\perp}$ up to $\approx 2 J_{\|}$(see Fig. 3). One obvious way to reduce $\Delta$ with respect to the maximum is to increase the ratio $J_{\|} / J_{\perp}$, in this case to $\gtrsim 1.5$. Such a large value is difficult to reconcile with the microscopic parameters, in particular with the similar $\mathrm{Cu}-\mathrm{O}$ bond lengths along the legs and the rung which has provoked a controversial discussion.26 Our analysis shows that any values of $J_{\|} / J_{\perp}$ larger than 1.2 can be excluded. Recently, it was pointed out that the neutron data are also consistent with an isotropic exchange $J_{\|} / J_{\perp} \approx 1-1.1$ and $J_{\|} \approx 900 \mathrm{~cm}^{-1}$ if a ring exchange of $\approx 0.15 J_{\|}$is taken into account 13 Consid- 
ering the fact that the ring exchange will renormalize $J_{\|}$ and $J_{\perp}$, this is in perfect agreement with our findings.

Judging the weight of the high-energy continuum one should note that it increases by taking into account an admixture of an isotropic form factor to the $\sin ^{4}(k / 2)$ form factor used here. Second, the 2-particle excitations considered here carry only $\approx 75 \%$ of the total spectral weight. In the $2 \mathrm{D}$ cuprates, Lorenzana et al. 31 argued that ring exchange increases the spectral weight at high energies. It will be most interesting to check the influence of ring exchange on the continuum of the ladders, since these provide a technically much better controlled ground. Note that the theoretical curves in Fig. 2 describe the frequency range of continuum absorption very well, which gives strong support to our interpretation.

Finally, we address the similarity of $\sigma(\omega)$ of the ladder $(\mathrm{Ca}, \mathrm{La})_{14} \mathrm{Cu}_{24} \mathrm{O}_{41}$ and of the undoped 2D cuprates (see Fig. 11). The 2D case does not show a truly bound state, the sharp peak at $2800 \mathrm{~cm}^{-1}$ in $\sigma(\omega)$ of $\mathrm{YBa}_{2} \mathrm{Cu}_{3} \mathrm{O}_{6}$ is caused by a resonance, an almost bound state lying within the continuum. 15 This main peak is well described in terms of 2-magnon-plus-phonon absorption, but the high-energy peak at $3800 \mathrm{~cm}^{-1}$ is absent in spin-wave theory. 1 Note that this discrepancy is particular for the 2D $S=1 / 2$ case, the high-energy excitations are absent in the comparable $S=1$ system $\mathrm{La}_{2} \mathrm{NiO}_{4}$ (Refs. 15, 32). In the cuprates, the magnetic origin of both peaks has been confirmed by absprption and Raman messurements under high pressure 33 It has been suggested that the highenergy weight in $2 \mathrm{D} S=1 / 2$ compounds is due to strong quantum fluctuations that go beyond spin-wave theory. The intriguing similarity of the 2-particle continuum of the $S=1 / 2$ quasi-1D ladder $(\mathrm{Ca}, \mathrm{La})_{14} \mathrm{Cu}_{24} \mathrm{O}_{41}$ with the $3800 \mathrm{~cm}^{-1}$ peak of $\mathrm{YBa}_{2} \mathrm{Cu}_{3} \mathrm{O}_{6}$ strongly indicates that the high-energy spectral weight is indeed a signature of strong quantum fluctuations in this compound.

In conclusion, the existence of the singlet bound state and the 2-particle continuum was demonstrated experimentally in the optical conductivity spectrum $\sigma(\omega)$ of the $S=1 / 2$ 2-leg ladder compound $(\mathrm{Ca}, \mathrm{La}){ }_{14} \mathrm{Cu}_{24} \mathrm{O}_{41}$. By two independent theoretical approaches we confirmed that the two sharp peaks plus the important broad continuum seen in experiment are the unambiguous signature of a $1 \mathrm{D}$ dispersive bound singlet with strong incoherent quantum fluctuations. The experimental spectral weight distribution reflects the theoretical selection rules perfectly. Quantitative analysis yields $J_{\|} \approx 1050-1100$ $\mathrm{cm}^{-1}$ and $J_{\|} / J_{\perp} \approx 1-1.1$. Our findings indicate that the similar experimental results for the high-energy excitations of the undoped high- $T_{c}$ materials are also due to strong quantum fluctuations.

We acknowledge fruitful discussions with E. MüllerHartmann. This project is supported by the DFG in FR 754/2-1, SP 1073 and SFB 484, by the BMBF 13N6918/1 and by the DAAD in the frame of PROCOPE.
${ }^{1}$ M. Grüninger et al., Phys. Rev. B 62, 12422 (2000).

${ }^{2}$ C.-M. Ho, V.N. Muthukumar, M. Ogata, and P.W. Anderson, Phys. Rev. Lett. 86, 1626 (2001).

${ }^{3}$ G. Aeppli et al., phys. stat. sol. b 215, 519 (1999).

${ }^{4}$ A.W. Sandvik and R.R.P. Singh, Phys. Rev. Lett. 86, 528 (2001).

${ }^{5}$ R.B. Laughlin, Phys. Rev. Lett. 79, 1726 (1997).

${ }^{6}$ E.M. McCarron et al., Mater. Res. Bull. 23, 1355 (1998); T. Siegrist et al., ibid., 1429.

${ }^{7}$ G.S. Uhrig and H.J. Schulz, Phys. Rev. B 54, R9624 (1996); erratum ibid. 58, 2900 (1998)

${ }^{8}$ K. Damle and S. Sachdev, Phys. Rev. B 57, 8307 (1998).

${ }^{9}$ O.P. Sushkov and V.N. Kotov, Phys. Rev. Lett. 81, 1941 (1998); V.N. Kotov, O.P. Sushkov, and R. Eder, Phys. Rev. B 59, 6266 (1999).

${ }^{10}$ C. Jurecka and W. Brenig, Phys. Rev. B 61, 14307 (2000).

${ }^{11}$ S. Trebst et al., Phys. Rev. Lett. 85, 4373 (2000).

${ }^{12} \mathrm{~W}$. Zheng et al., preprint (cond-mat/0010354).

13 M. Matsuda et al., Phys. Rev. B 62, 8903 (2000); J. Appl. Phys. 87, 6271 (2000).

${ }^{14}$ S. Sugai and M. Suzuki, phys. stat. sol. b 215, 653 (1999).

15 J. Lorenzana and G.A. Sawatzky, Phys. Rev. Lett. 74, 1867 (1995); Phys. Rev. B 52, 9576 (1995).

${ }^{16}$ P. Jordan and E. Wigner, Z. Phys. 47, 631 (1928).

${ }^{17}$ F.J. Wegner, Ann. Phys. 3, 77 (1994).

18 C. Knetter and G.S. Uhrig, Eur. Phys. J B 13, 209 (2000).

19 U. Ammerahl and A. Revcolevschi, J. Crystal Growth 197, 825 (1999); U. Ammerahl, PhD thesis, Univ. of Cologne, 2000.

${ }^{20}$ N. Nücker et al., Phys. Rev. B 62, 14384 (2000).

${ }^{21}$ Y. Mizuno, T. Tohyama, and S. Maekawa, J. Phys. Soc. Jpn. 66, 937 (1997).

${ }^{22}$ S.A. Carter et al., Phys. Rev. Lett. 77, 1378 (1996).

23 The transmission was measured on samples with a thickness of $44 \mu \mathrm{m}(x=5)$ and $60 \mu \mathrm{m}(x=4)$.

${ }^{24}$ Both methods are controlled in the sense that they become exact on $J_{\|} / J_{\perp} \rightarrow 0$.

${ }^{25}$ H. Kleinert, Path Integrals in Quantum Mechanics, Statistics, and Polymer Physics, World Scientific (1995)

${ }^{26}$ D.C. Johnston et al., preprint (cond-mat/0001147) and references therein.

27 Z. Weihong, V. Kotov, and J. Oitmaa, Phys. Rev. B 57, 11439 (1998).

${ }^{28}$ J. Lorenzana and R. Eder, Phys. Rev. B 55, R3358 (1997).

${ }^{29}$ The precise mixture depends on the $3 \mathrm{D}$ phonons and is beyond the physics of an isolated ladder. However, it does not affect the peak frequencies.

${ }^{30}$ Energies can be extrapolated much more accurately than spectral densities.

31 J. Lorenzana, J. Eroles, and S. Sorella, Phys. Rev. Lett. 83, 5122 (1999).

32 J.D. Perkins et al., Phys. Rev. B 52, R9863 (1995).

${ }^{33}$ V.V. Struzhkin et al., Phys. Rev. B 62, 3895 (2000). 